\title{
POST-REFLECTING ON THE PROCESS OF INTEGRAL DESIGN OF AN ADAPTIVE FOOTBRIDGE STRUCTURE USING BENDING-ACTIVE PRINCIPLES
}

\author{
M.C. PHOCAS, O. KONTOVOURKIS \& K. ALEXANDROU \\ Department of Architecture, Faculty of Engineering, University of Cyprus, Cyprus.
}

\begin{abstract}
Contemporary design approaches of adaptive structures enhanced to a great extent through digital technology, gradually acknowledge the fact that the area encompasses a number of disciplines, bringing together a number of distinct modes of investigation. Within this frame, the interactive development of a cable bending-active footbridge structure presented in the current paper aims at clarifying the process of integral design applied. The structural prototype consists of two parallel series of bendingactive PETG members with initial inverted curvatures forming continuous elastic curvilinear elements, which are horizontally interconnected through cables. In a preliminary design stage, the structure is conceptualized through cyclically iterated physical modelling and preliminary finite element analysis. The design development stage is based on digital simulation, whereas the load-bearing and adaptive behaviour of the structure is examined and visualized in real time according to the pretension of the cables and predefined pedestrian movement scenarios, respectively. Following the construction design and manufacturing of the structural members, the design evaluation stage addresses beyond verification issues of the design proposed, structural optimization aspects through investigation of suitable pretension values of the cables and geometric characteristics of the bending-active members. The integral design approach of the adaptive structure is exemplary for integrating different modes of operation and digital investigation tools in achieving effective load-bearing characteristics and adaptability of the structure.
\end{abstract}

Keywords: adaptive structures, bending-active members, hybrid systems, integral design, interdisciplinary design.

\section{INTRODUCTION}

Contemporary design approaches of adaptive structures enhanced to a great extent through digital technology, gradually acknowledging the fact that the area encompasses a number of disciplines, bringing together a number of distinct modes of research and types of knowledge. Research into adaptive structures is becoming conscious of these interactions and of the particular need to be further nonlinear and integrative across disciplinary boundaries. The design process itself provides possibilities for interdisciplinary research, ranging from the conceptual design to the development in detail, optimization and fabrication. This might be interpreted as the conceptual, refined and detailed stage of an integral methodology applied in the design process, where 'design generation' and 'evaluation' stages are acting in a feedback loop manner [1]. In this respect, nonlinear design processes based on interdisciplinary performance optimization criteria are considered to be crucial, leading to more or less interactive, iterative closed-loop design processes. Hence, digital design and numerical analysis may be applied throughout different stages of the design process shaping individual iterative operational circles that are linearly linked to previous and subsequent ones. Within this frame, the use of advance digital tools in analysis and simulations leads towards design possibilities and adaptive structural transformation behaviour of morphological outcomes at digital and physical level [2]. At the same time, investigation of the structures kinematics in real time 
through visualization of results is considered to be a significant and indispensable aspect of the design process.

Addressing the fact that kinetic bar structures with embedded control systems have found to be technologically expensive presupposing high-end technological principles [3,4], new soft materials in architecture are recently investigated [5]. Such materials may constitute mechanisms with autonomous, adaptive physical behaviour. Compared to technical linkage systems, bending-active mechanisms replace local hinges by elastic deformations of their members and thus distribute the acting forces over a wider area in which bending takes place. This alternative approach renders the possibility to form complex single- or double-curved primary structures from straight or planar members. Sufficiently thin component thickness thereby allows for small bending radii and thus results in low bending stresses. The application of bending-active members has been recently demonstrated with a pavilion prototype construction with birch plywood lamellas at the University of Stuttgart in 2010 [6] and kinetic shading elements for the Biomimetic Media Façade of the Thematic Pavilion at Expo 2012 in Yeosu, South Korea [7].

In extent, for optimized resistance to time-varying external forces, structural rigidity can be increased either by combination of bending and pretension of individual elements, or by coupling of multiple elements to a hybrid system [8]. The use of bending principles in hybrid systems enables initial complex geometries, subsequent stabilization through additional pretension, reversible deformability and sustainable controlled kinematics of the members. The crane model originally proposed by Frei Otto [9], of a central flexible curved element that may obtain different configurations through three sets of longitudinal cables, provides such an adaptive hybrid bending-active system. Another example is the hybrid prototype structure of three polyethylene terephthalate glycol (PETG) lamellas interconnected by struts with variable length [10]. Further developments of adaptive hybrid structures, consisting of primary bending-active members and a secondary system of struts and cables with closed circuit and variable length, have been proposed in $[2,4]$. In these prototype developments, the cables have a dual function: to stabilize the primary members and to provide the structure with controlled transformability for obtaining different configuration states throughout the adaptation process.

The integral design of an adaptive footbridge structural prototype presented in the current paper reflexively addresses the above-mentioned considerations with regard to the design process involving structural lightweight and modularity, natural systems inspired adaptability derived from elastic material properties and controllability of stiffness and shape. The integral design of the adaptive structure is reflected in interactive phases of development following a performance-based bottom-up approach. These interactive phases consist of the preliminary design based on physical modelling and initial numerical analysis, the design development and evaluation through digital and numerical analyses with regard to different geometrical and mechanical characteristics of the structural members.

\section{INTEGRAL DESIGN PROCESS}

The role of digital design and computation in the conceptualization and design development up to date is indicative for a new syntax of emerging interdisciplinary design. Though not new to other design industries, computational platforms of operation and real-time performance simulators provide meanwhile robust visualization and feedback features that can be associated with geometrical digital design models. Design developments at various stages encompass further parametric investigations with regard to form, material and structure [11]. 
In this respect, a diagrammatic framework of an open-loop performance-based design has been proposed in [12], supporting the development from concept to detail following re-evaluations within a performative context in a nonlinear way, i.e. by moving from the conceptual to the detailed design phase and vice versa. In parallel, interactivity within the decision-making process may provide a promising mode of operation.

The development of bending-active structures may lie within a similar frame of investigation, whereas decisive criteria might include the length and surface dimension of the primary members and the simulation of material behaviour. Form-finding in bending-active structures is largely influenced by the geometrical dimensions of the primary members that undergo bending deformations as a result of the constraining boundary conditions, as well as their mechanical behaviour. Thereby the development of bending-active structures entails noticeable complexities in the design process for satisfying both architectural specifications and mechanical behaviour, and may be achieved through a combination and integration of various modelling techniques that include physical, digital modelling and computational finite element analysis [5]. In this frame, application of active bending principles to integral structural concepts may initially result from a behaviour or geometry-based design approach. In a behaviour-based approach, bending is initially used intuitively; the system's geometry and structural behaviour is studied empirically. Material limitations are tested physically. In a geometry-based approach, the system's geometry is predefined based on analytical geometry or experimental form-finding methods, both of which are used as controlled means to approximate the actual bending geometry. Material limitations are considered analytically. Furthermore, in an integral approach the elastic bending deformation is analysed through numerical form-finding, which enables full control of material behaviour-based geometry. Material characteristics and limitations are included in the numerical analysis model. The present work seeks to integrate major parts of these approaches through iterative development of physical modelling, simulation techniques and numerical analysis.

In the preliminary phase of the prototype system's conception, physical small-scale models are developed and investigated aiming at the structures material behaviour under different loading conditions. To achieve this, the process of the design development follows a feedback loop methodology and is integrated with the process of the evaluation of results. In this loop, the 'preliminary design' investigation introduces physical prototypes and includes initial numerical analysis. The 'design development' stage contains parametric associative design tools including physics-based simulations, and the construction design of the members and connections. The 'design evaluation' stage involves mechanisms of verification of the proposed solutions through finite element structural analysis. The integral design process followed in the conception, development and evaluation of the prototype structure relies thus on physical, virtual representation of morphology and behaviour and computational foundation that open up new possibilities in the design investigation allowing real-time interactive morphological and behavioural transformation. While the preliminary investigation phase offers in parallel to the conception, an understanding of the structures design and behaviour potentialities, the design development leads to an understanding of the local and global structural behaviour and the formulation of the structural components, and the design evaluation enables further closed-loop optimization processes with regard to the structural performance. The suggested feedback loop platform is considered as an interactive form of design assistance building upon traditional quantitative analytical and real-time simulation techniques within the design process of adaptive structures. In this manner, the cyclical iterative process allows simultaneous design exploration and verification of results within an integral context. 


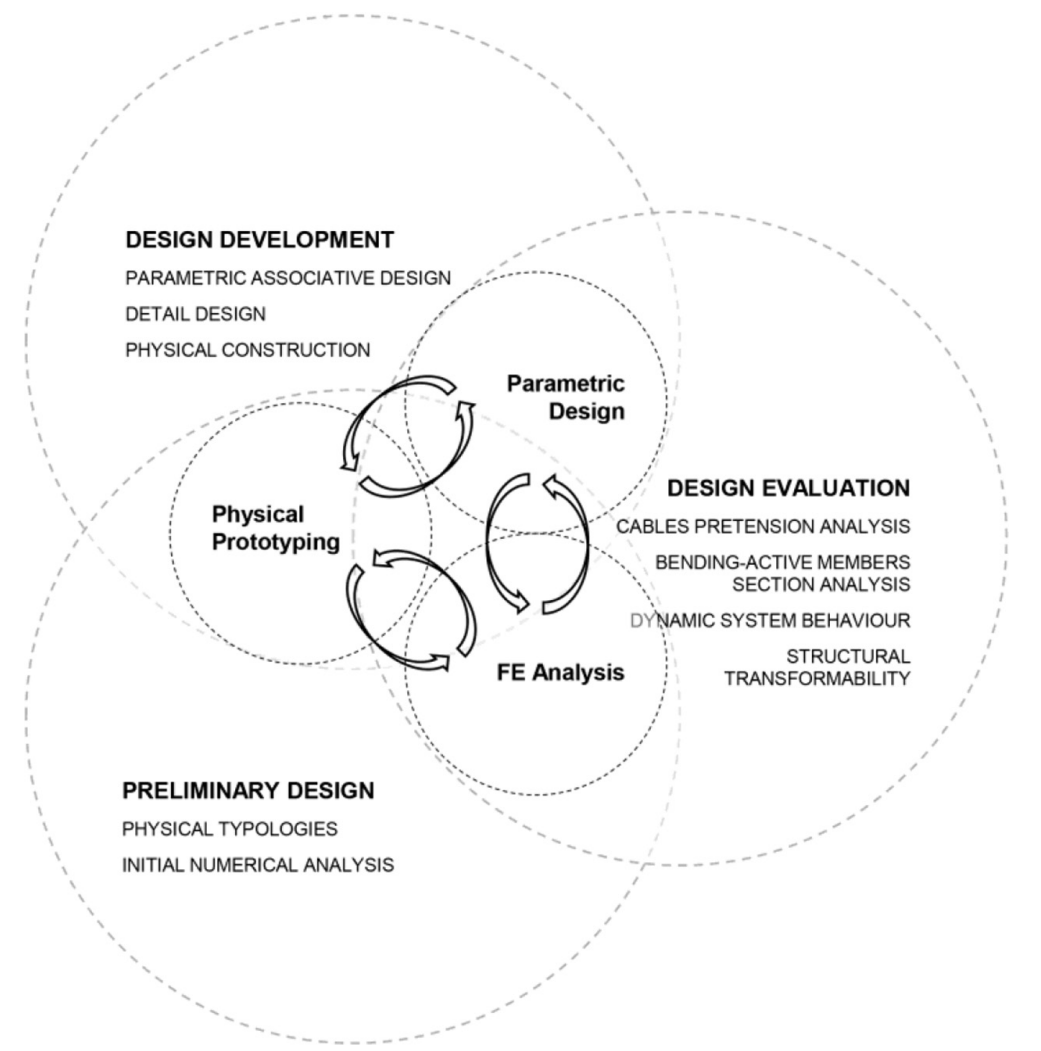

Figure 1: Feedback loop methodology followed in the integral design process.

Figure 1 describes the design methodology applied for the development of the structural prototype, which consists of three main integrated investigation platforms, i.e. physical prototyping, parametric design and finite element analysis, all interrelated in pairs in formulating the overall development steps.

\section{PRELIMINARY DESIGN}

Physical form-finding of the structure is applied in the preliminary design stage, to respond to certain architectural intentions of the project, develop the systems typology and explore topological data for providing initial understanding of its geometric, mechanical and kinetic behaviour through experimentation [13]. Initial conceptions with physical models allow for an instantaneous and intuitive change of the input variables to an immediate form-finding result. This synchronicity offers flexibility in the initial design process, while physical form-finding yet stays within mechanical feasibility. In the creative process of building physical models, basic principles for short-cutting forces and coupling smaller units into larger structural systems are easily unveiled due to an instant mechanical feedback from the model. The process, often unintuitive due to the complexity of form-force relationships, enables exploration of diversity in form. Once a primary system is developed, variation of length and surface dimensions allows fine-tuning of geometry and structural interaction. In particular, substantial for the exploration of the bending-active structure is the fact that the geometry of 
uniform singular elements is independent of material behaviour and scale. Comparative physical form-finding experiments may be, therefore, initially carried out at various scales. The topological data provided here enable to explore more precise geometric and mechanical behaviour. Iterations between physical experimentations and numerical analysis allow intermediate realizations and decisions with regard to composition, load-bearing and kinematics of the system.

\subsection{Physical typologies and initial numerical analysis}

In structural configurations initially investigated through physical models, the primary elements consist of pairs of continuous PETG lamellas with dimensions of 700/25/2 mm, joined at nine points and bended to form continuous elastic curvilinear elements (Fig. 2). In the first two alternatives, eight units are stabilized at mid-span through struts. The kinematics of the systems is induced by cables with closed circuit and variable length, passing through the units at the perimeters. In a third alternative, units of 700/25/2 and 900/25/2 mm dimensions for the primary and secondary lamellas, respectively, are only stabilized through a cable with closed circuit and variable length, passing through at mid-height of the upper lamella. In a final alternative, the stabilization of the units is achieved with short length cables interconnected at all unit points. The kinematics of the system is enabled through respective length adjustments of the cables at the respective segments. Although the system consists of a higher number of secondary members and connections, the segmentation of the tension-only members enables higher controllability in its kinematics, compared to the previous mentioned systems.

The selected system configuration for a two hinge-supported girder consists of pairs of inversely curvilinear PETG lamellas resulting in eight hinge connected rhomboid units. The units are stabilized by rows of three horizontal cable pairs at their respective upper, middle and lower points. The units have $1.375 \mathrm{~m}$ axial length and $0.5 \mathrm{~m}$ height. The flat rectangular sections used have dimensions of $250 / 20 \mathrm{~mm}$ and the cables, a diameter of $20 \mathrm{~mm}$. The total span of the structure amounts to $11 \mathrm{~m}$. The structural system has been compared in its load-bearing behaviour to three similar systems with the same overall span: a pantograph structure of scissor elements, a hybrid system of rigidly connected beams strengthened by struts and diagonal cables, and a hybrid system of hinge-connected beams strengthened by struts, horizontal and diagonal cables [13]. The physical models of the structural systems have been investigated with the finite element software program SAP2000 based on a vertical uniform load of $2.5 \mathrm{kN} / \mathrm{m}(\mathrm{Q})$. At this stage, in the two-dimensional nonlinear analysis, the
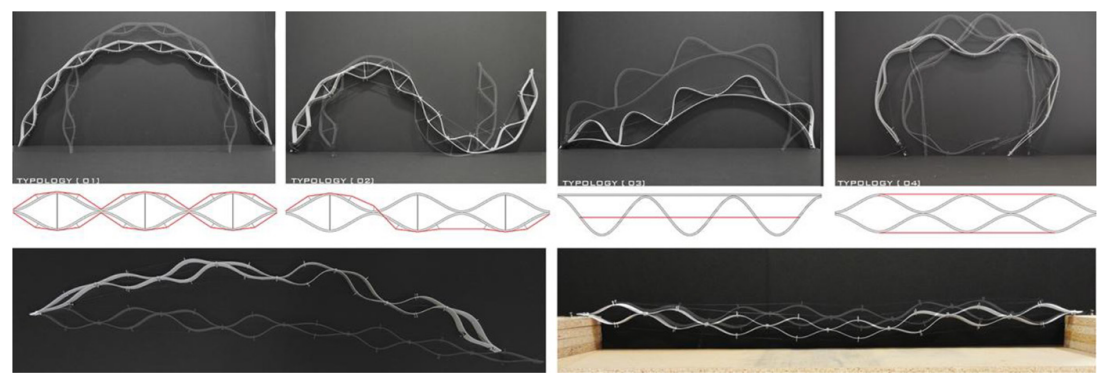

Figure 2: Configuration case studies of adaptive cable bending-active structure. 
cables have been modelled as frame objects with zero compression limit to represent the behaviour of flexible tension-only members. The maximum vertical deformation of the system amounts to $14.41 \mathrm{~cm}$, i.e. $1.31 \%$ of the overall span. A comparison of the systems load-bearing behaviour is included in [13]. In extent, certain advantages sought after in the cable bending-active structure that was further investigated, refer to the material deformability that would enable reversible deformations with relatively low bending stresses and, therefore, a high degree of geometrical transformability in its kinematics.

\section{DESIGN DEVELOPMENT}

In the design development stage, digital simulation is introduced aiming at first place at providing correlations between the physical and the digital model using parametric associative design logic. This investigation input is considered to be significant, while focussing on the adaptive structural behaviour induced by the behaviour of the elastic material of the primary structural members. The use of parametric associative tools in the simulation procedure allows preliminary investigation and initial insight into the adaptive behaviour of the structure with regard to its geometrical transformability and actual physical behaviour. The investigation outcomes comprise integral iterative parts of the structural performance verification described in the next section. For the preliminary simulation of the adaptive structure, the parametric design software Grasshopper and the live physics software Kangaroo (plug-in for Rhino and plug-in for Grasshopper, respectively) were used.

Following digital conception of the structure, the construction design of the members leads to further development of the members' geometry and connections and verification of the load paths within the structure (Fig. 3). Equally important is the fact that the construction design of the structural members and connections itself should enable, at this stage, minimum self-weight, standardization and mass customization, as well as ease of fabrication, assembly and erection [14]. The detail design and physical construction of the structure in small scale are documented in [13].

\subsection{Parametric associative design}

In the simulation procedure, the initial geometrical configuration, the parametric associative control, as well as the physical properties of individual structural members that constitute the overall structural behaviour, were taken into consideration. In terms of geometrical and physics-based parametric control and behaviour, the structure was initially examined at local level focusing on the curvilinear cable bending-active structure, and then at global level investigating the geometrical definition and behaviour of the overall footbridge structure. To achieve

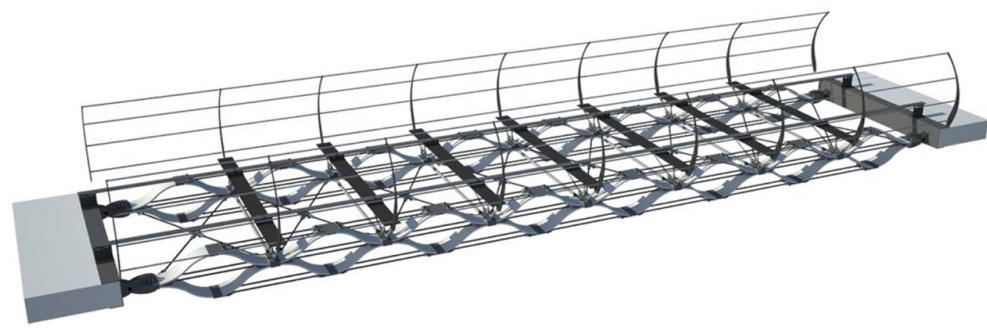

Figure 3: Three-dimensional structure. 
this, the structural members are divided into two main categories according to their physical behaviour; the PETG elastic lamellas acting as bending-active members and the segments of the horizontal cables acting as tension-only members. These are associatively related and physically encoded using at this stage, a number of approximate physics-based forces including bending, pretension, gravity (self-loading) and movable vertical point loading. The resultant force acting upon the cable bending-active structure accelerates the structural behaviour simulation.

The PETG lamellas and their elasticity were digitally simulated using bending behaviour rules, based among other approaches, on the idea of three defined consecutive points in a discretized structural member [15]. Analytically, the procedure involves the design definition of stripes, in this case, in the form of sine-like curves, and their parametric division into segments and points. Given that any three consecutive points on a curve that are deviated tend to be aligned on a straight line, this behaviour accelerates the actual-like bending of the structural members. To keep the pair of lamellas in their initial bending position, the middle cables are parametrically defined as axial members, controlled and simulated by applying spring behaviour on the basis of Hooke's Law of elastic stress-strain behaviour [16,17]. The spring behaviour is primary defined through the parametric control of the spring length that is the metric control of the input 'rest length' in relation to the input 'start length' influencing the members under tension or compression. If the 'rest length' is equal to the 'start length', then the member is considered to exhibit elastic behavior. If the 'rest length' is smaller than the 'start length', then the member is under tension. In this case, axial cables are parametrically connected with pairs of bending-active members in the middle articulated joints of the structural units, providing an overall behaviour that involves bending and tension. Moreover, the bending-active members are extended in two dimensions by defining the width. The generated surface is broken down into triangles, and edges are physically encoded by applying spring behaviour aiming to stabilize and achieve rigidity of the two-dimensional lamellas. Main investigations conducted through associative digital modelling refer to the variation of the cables pretension values and the pedestrian loading. The investigations aim at revealing qualitatively the local and overall structural load-bearing behaviour and transformability. These are included in the next section, with the respective iteratively succeeding numerical evaluations.

\section{DESIGN EVALUATION}

The design process applied for the structural prototype development exhibits iterative integration of finite element analysis at various levels. Throughout the design process, two phases of creativity have proved to be feasible. The first is situated at the beginning of the process that mostly used physical modelling with parallel, initial numerical analysis for providing verifications of intermediate observations. This preliminary stage served as a quick but materially informed method of funnel management to create the basis of a physically feasibly system from many possibilities. The second phase lies between the form-finding and the structural design phase, where adaptations and specifications in the design have been pursued through associative digital modelling of the system, succeeded by the detail design and physical construction phase. At this intersection, finite element analysis processed information between form-finding and structural design and thereby became part of the actual design, rather than a mirroring instrument that verifies the feasibility of decisions taken in other modelling environments. Numerical analysis integration in the creative process of the design methodology requires a setup of bidirectional information flow to enable fast iterations in the 
creative design phases. The analysis of the structure refers primarily to suitable pretension values for the cable members in reducing the maximum deformations at mid-span. Further investigations of the structural behaviour for a selected cables pretension case, with regard to variation of the section thickness of the bending-active members, and the systems dynamic behaviour are included in [13]. Finally, in the current section, the structures transformability is investigated parametrically in associative digital environment for different pedestrians' movement scenarios and then a selected one is numerically evaluated. Possible directions towards optimization introduced through numerical structural analysis prove to be significant in the design evaluation stage. Such iterative steps of design verification and optimization constitute nonlinear loops in the interdisciplinary design process, influencing other design stages as well.

\subsection{Cables pretension analysis}

Initial investigation of the cable bending-active structure load-bearing behaviour has been based on the cables length and their pretension values. The cables, parametrically defined as axial spring elements, allow simulation of the pretention effect by setting the 'rest length' value lower than the 'start length'. In the specific case example, the start length was set to $1375 \mathrm{~mm}$. The 'spring stiffness', which defines the axial stiffness of spring elements, has been set to a default value of 4000 for all cable rows. The pretension level of the upper and middle cables has been set to zero, i.e. rest length equal to start length. Under a uniform distribution of vertical load (unary force), the pretension level of the lower cables has been readjusted into multiple sets of values, aiming at reducing the maximum vertical deformations of the system at mid-span (Fig. 4). The linear shortening of the spring elements has effectively deformed the initial geometry of the bending-active elements, inducing at the

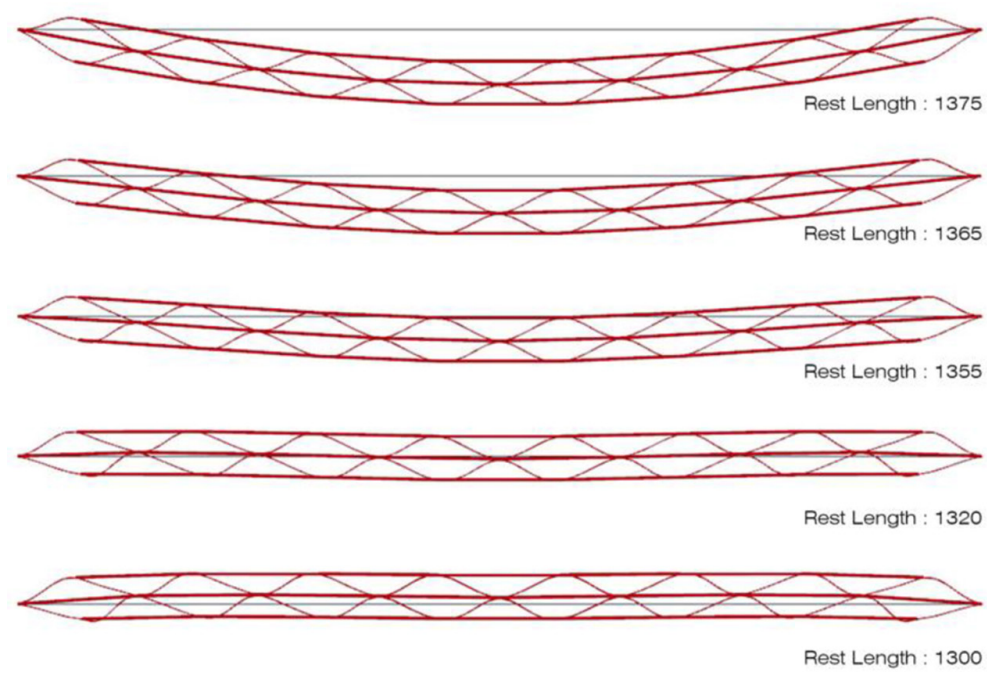

Upper and Middle Cable Rest Length : 1375

Cable Stiffness : 4000

Unary Force : -250

Figure 4: Parametric associative system deformation behaviour due to lower cables pretension variation. 
same time certain relative deformations of the rhomboid units. The lower mid-points of each unit have been displaced towards the centre of the structure and the upper mid-points towards the supports.

In the design evaluation stage, optimization of the cable bending-active structure is primarily based on the shape design of the cables and their pretension. The cables have been assigned with different pretension values for providing improved structural stabilization under external loading and maximum vertical deformations reduction at mid-span under a vertical uniform load of $2.5 \mathrm{kN} / \mathrm{m}(\mathrm{Q})$. Cable elements were used to capture both the catenary nature of the tension-only members and their geometrical nonlinear characteristic behaviour. Initially, suitable selection of the respective target pretension force in the cables neutralizes the structures bending deformation at mid-span with regard to its self-weight. The initial tension forces in the cables are associated with the shape of the members, whereas the upper, middle and lower cables obtained maximum values of approximately 3, 25 and $45 \mathrm{kN}$, respectively. The pretension values of the middle and lower cables have been independently differentiated for each cable row, to cover a range of $10-220 \mathrm{kN}$ target pretension forces, while keeping the pretension values in the other tension-only member rows constant [13].

The maximum absolute system responses for the uniform vertical loading considered in the numerical nonlinear analyses under different pretension values of the lower cables are included in Table 1. A certain divergence of $21.62 \%$ and $11.24 \%$ with regard to the maximum axial force in the lower cables and the vertical deformations of the structure, respectively, are registered by modelling the tension-only members as cable elements without pretension compared to the initial modelling of the elements as frame objects with zero compression limit at the preliminary design stage. This is attributed to the fact that the nonlinear analysis with cable elements includes both tension-stiffening and large deflections nonlinearity in the members' response. In principle, the lower cables seem to control effectively the deformations of the system. An increase of up to $200 \mathrm{kN}$ target pretension forces in the lower cables, and constant pretension of 10 and $20 \mathrm{kN}$ of the upper and middle cables, respectively, succeeds in practically neutralizing the maximum deformations of the system at mid-span. This is accompanied by an increase of $288 \%$ and $225 \%$ of the axial forces and bending moments in the primary members, respectively. Further pretension of the lower cables induces inverse

Table 1: Maximum absolute cable bending-active system responses for uniform vertical loading $\mathrm{Q}$ and different pretension values of the cables.

\begin{tabular}{|c|c|c|c|c|c|c|c|}
\hline \multirow{2}{*}{$\begin{array}{l}\text { Cables } \\
\text { pretension } \\
\text { Case }\end{array}$} & \multicolumn{3}{|c|}{ Primary members } & \multicolumn{3}{|c|}{ Tension-only members } & \multirow[b]{2}{*}{$\mathrm{U}(\mathrm{cm})$} \\
\hline & $\mathrm{N}(\mathrm{kN})$ & $\begin{array}{l}\mathrm{Q} \\
(\mathrm{kN})\end{array}$ & $\begin{array}{l}\mathrm{M} \\
(\mathrm{kNm})\end{array}$ & $\begin{array}{l}\text { Upper } \\
\mathrm{N}(\mathrm{kN})\end{array}$ & $\begin{array}{l}\text { Middle } \\
\mathrm{N}(\mathrm{kN})\end{array}$ & $\begin{array}{l}\text { Lower } \\
\mathrm{N}(\mathrm{kN})\end{array}$ & \\
\hline $0-0-0$ & 53.36 & 20.97 & 2.40 & 8.74 & 84.31 & 147.66 & 12.79 \\
\hline $10-20-40$ & 90.49 & 35.06 & 3.84 & 24.67 & 143.97 & 249.16 & 14.80 \\
\hline $10-20-80$ & 103.46 & 39.62 & 4.16 & 41.79 & 140.20 & 267.77 & 10.05 \\
\hline $10-20-150$ & 131.23 & 49.38 & 4.84 & 80.56 & 152.02 & 337.65 & 4.16 \\
\hline 10-20-190 & 149.48 & 55.81 & 5.28 & 105.98 & 167.23 & 391.58 & 1.30 \\
\hline $10-20-200$ & 154.14 & 57.45 & 5.40 & 112.35 & 171.53 & 405.36 & 1.89 \\
\hline $10-20-220$ & 163.54 & 60.77 & 5.63 & 124.98 & 180.88 & 433.37 & 3.41 \\
\hline
\end{tabular}


deformations of the system. The analyses conducted based on a differentiation of the middle cables pretension levels indicated no substantial influence on the internal forces of the members. In these cases, the maximum vertical deformation of the structure decreases only to approximately $14.5 \%$ for a respective pretension increase of the middle cables from 40 to $150 \mathrm{kN}$ and a constant pretension of $10 \mathrm{kN}$ of the remaining tension-only members. The analysis results suggest a target pretension force of 10,20 and $190 \mathrm{kN}$, to be applied in the upper, middle and lower cables of the system, respectively, i.e. pretension case: 10-20-190. The selected pretension level reaches approximately $43 \%$ of the maximum elastic tensile stress of $140 \mathrm{kN} / \mathrm{cm}^{2}$ of the lower tension-only members with a section diameter of $20 \mathrm{~mm}$. Furthermore, the system responses suggest that an additional target pretension force in the lower cables of up to $30 \mathrm{kN}$ could be provided by an active control system, where limitation of the vertical deformations of the structure under excessive moving loads is necessary.

An optimization of the system behaviour may be achieved through differentiation of the lower individual cables pretension. This would also enable further investigation and understanding of the local and overall structural load-bearing behaviour. By referencing the cables pretension case of 10-20-190, two possible optimization paths are explained among numerous respective possibilities available. In all cases, the objective is to minimize the structures overall deformation at mid-span, and at the same time to keep low and symmetrical the relative deformations of the rhomboid units, i.e. difference between upper and lower unit bending-active members deformations at their mid-points. The latter criterion is especially significant for the outer units that exhibit the high non-symmetrical relative deformations due to the anchorage of the outer cables at the supports.

In the first optimization case followed, the pretension of the outer lower cables is initially decreased to $150 \mathrm{kN}$. The outer units relative deformations increase from 0.19 to $0.55 \mathrm{~cm}$ and the structures deformation at mid-span from 1.30 to $1.51 \mathrm{~cm}$. In achieving reduction of the outer units relative deformations, the pretension values are further decreased to $100 \mathrm{kN}$. The outer units relative deformations amount to $0.06 \mathrm{~cm}$ and the structures deformation at midspan $1.81 \mathrm{~cm}$. Subsequently, the middle cables pretension is stepwise increased, up to a value of $300 \mathrm{kN}$. In this case, the outer units relative deformations increase to $0.57 \mathrm{~cm}$ and the structures deformation at mid-span decreases to $0.96 \mathrm{~cm}$. All nodal intersection points of the units at mid-height have positive displacement values. Further increase of the lower cables pretension at mid-span yields negative displacements of the first and second nodal points on each side of the system. Therefore, the pretension of the cables adjacent to the outer ones is further reduced, so that respective differences are smoothened out. For these cables, a pretension value of $150 \mathrm{kN}$ is selected and deformation values of 0.50 and $1.26 \mathrm{~cm}$ for the outer units and the system at mid-span are obtained. An increase of the pretension of the cables adjacent to the middle ones, to $220 \mathrm{kN}$, yields respective response values of 0.58 and $0.91 \mathrm{~cm}$. Further increase of the cables pretension initiates negative displacements of the respective units' nodal points from above and at mid-span. Therefore, the preferable pretension case for the lower cables would be 100-150-220-300-300-220-150-100.

In the second optimization case example, the pretension is only differentiated between adjacent lower cable pairs. Initially, the outer cable pairs pretension is decreased to $150 \mathrm{kN}$. The outer units relative deformations decrease from 0.19 to $0.11 \mathrm{~cm}$ and the structures deformation at mid-span, from 1.30 to $0.52 \mathrm{~cm}$. Due to negative deformations of the outer unit pairs, further increase of the pretension of the inner cable pairs, up to $230 \mathrm{kN}$, is applied. In this case, the outer units relative deformations increase to $0.56 \mathrm{~cm}$ and the structures deformation at mid-span decreases to $1.05 \mathrm{~cm}$. Negative deformations develop only in the outer 
units. Further increase of the pretension in the outer cable pairs does not improve the systems deformation behaviour towards a uniform shape. In this respect, a slight improvement may be achieved through increase of the outer cable pairs pretension to $160 \mathrm{kN}$ and decrease of the inner cable pairs pretension to $220 \mathrm{kN}$. This case leads to respective response values of 0.58 and $1.10 \mathrm{~cm}$, and to negative displacements of the outer nodal points only of $0.45 \mathrm{~cm}$. Taking into consideration the amount of pretension required to achieve the optimization objective, the pretension case 160-160-220-220-220-220-160-160 would be of preference among the ones investigated in both optimization case examples.

\subsection{Structural transformability}

Adaptation behaviour with regard to external human related influences was initially examined in the associative digital environment by parametric control of individual pedestrian positions, while moving with constant speed from left to the right [17]. The digital simulation of the three-dimensional structure is achieved by positioning at a certain parallel distance the two pairs of stripes following similar physics-based behavioural principles. The cable bending-active structures are stabilized by using structural members working in compression and tension and by connecting them diagonally at each articulated joint of the units. Their behaviour is simulated using spring forces with equal 'start' and 'rest length'. To avoid torsion of the elastic stripes, hinge behaviour is introduced aiming to avoid bending between the twodimensional virtual plane of stripes and of the axial members, i.e. acting for diaphragmatic purposes. The bending-active behaviour of the three-dimensional load-bearing structure was investigated based on the motion of pedestrians with different movement directions, positions in space and time of activation (Fig. 5).

In all scenarios, point loading is distributed uniformly to the pair of cable bending-active structures and the force magnitude is proportional to the distance between the two consecutive articulated joints of the primary structures. For simulation purposes, point loading influences only each pair of adjacent articulated joints of the structure. With regard to scenario 01 , geometrical transformation stages show the structures passive behaviour in discrete time steps, when a pedestrian is moving across the footbridge with constant speed. In scenario 02 , bending-active behaviour is examined by demonstrating various geometrical transformation possibilities influenced by two external point loads; one movable and one static that is represented by a pedestrian moving across the footbridge with constant speed and a second pedestrian standing in the middle of the structure, respectively. The complexity of the movement scenarios is increased in scenario 03, where two pedestrians are moving across the footbridge, in different directions and with same constant speed, while at the same time a third pedestrian is standing at a randomly selected position on the deck. Although the position of pedestrians in each time step allows the examination of the structures behaviour under the influence of randomly distributed point loads, the cable bending-active structures behaviour and its curvilinear deformation indicate the effective collaboration of the primary with the secondary members. Finally, in scenario 04, a pedestrian is moving across the footbridge with constant speed, while two other pedestrians are standing on the deck at same random position, of $2 / 3$ of the overall span. In this scenario, in parallel to the bending-active behaviour of the elastic lamellas, the active control possibility of the structural members is examined. The control concept would activate the lower cables, parametrically simulated by modifying the 'rest length' of the spring behaviour. A 'rest length' value close to the 'start length' value shows the gradual change of the cable length and hence its behaviour from 


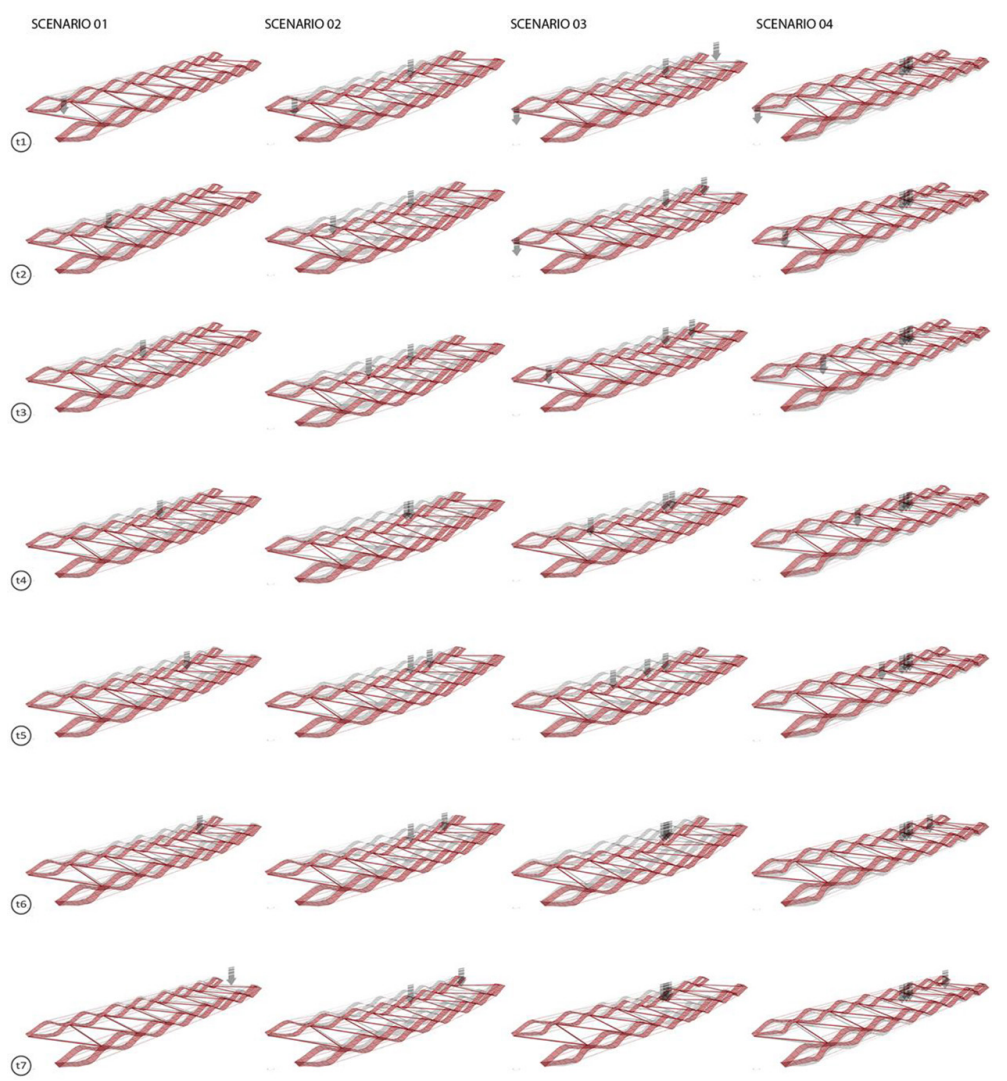

Figure 5: Simulation of structure transformability for different pedestrian movement scenarios.

tension to compression enabled through its pretension. This can be controlled by multiplying the 'start length' with a value from 0 to 1 . The simulation of the bending-active members together with an approximate at this stage, pretension of the cables and the compression members behaviour proves the ability of the footbridge structure to be stabilized and reach an equilibrium position in each time step.

The load-bearing behaviour of the selected structure with cables pretension of 10-20-190 is numerically investigated according to the previously digitally examined pedestrians movement scenario 04. Each pedestrian is represented by an additional point load of $8 \mathrm{kN}$ to the uniform distributed loading of $2.5 \mathrm{kN} / \mathrm{m}$ already considered in the analyses. All loads divided in half are considered as external loads acting on couples of adjacent joints, respectively. The loading conditions of the structure comply with the geometric positioning of the pedestrians in the discrete digital movement scenario. Therefore, the comparative analysis contains seven different loading cases of the structure, whereas only the initial one consists of the standing pedestrians' loads. Primary aim of the analysis is to investigate the responsiveness of the structure through its relative deformations in each loading case, induced by the respective loads of the pedestrians. The results obtained with regard to the maximum internal forces of the members and relative deformations are included in Table 2 . The analysis proves, at first place, the significance of the lower cables with regard to the systems responsiveness. All 
Table 2: Maximum absolute cable bending-active system responses for uniform vertical loading Q and pedestrians loading according to movement scenario 04 .

\begin{tabular}{|c|c|c|c|c|c|c|c|}
\hline \multirow[b]{2}{*}{$\begin{array}{l}\text { Loading } \\
\text { case }\end{array}$} & \multicolumn{3}{|c|}{ Primary members } & \multicolumn{3}{|c|}{ Tension-only members } & \multirow[b]{2}{*}{$\begin{array}{l}\Delta \mathrm{U} \\
(\mathrm{cm})\end{array}$} \\
\hline & $\mathrm{N}(\mathrm{kN})$ & $\mathrm{Q}(\mathrm{kN})$ & $\mathrm{M}(\mathrm{kNm})$ & $\begin{array}{l}\text { Upper } \\
\mathrm{N}(\mathrm{kN})\end{array}$ & $\begin{array}{l}\text { Middle } \\
\mathrm{N}(\mathrm{kN})\end{array}$ & $\begin{array}{l}\text { Lower } \\
\mathrm{N}(\mathrm{kN})\end{array}$ & \\
\hline A & 413.50 & 56.45 & 3.75 & 122.60 & 194.40 & 515.60 & 5.38 \\
\hline $\mathrm{B}$ & 413.50 & 56.77 & 4.77 & 122.60 & 194.60 & 525.00 & 5.82 \\
\hline $\mathrm{C}$ & 413.60 & 59.00 & 4.71 & 122.50 & 194.70 & 534.50 & 6.21 \\
\hline $\mathrm{D}$ & 413.60 & 58.57 & 4.68 & 122.50 & 194.70 & 544.30 & 6.49 \\
\hline $\mathrm{E}$ & 413.62 & 58.13 & 4.65 & 122.60 & 194.70 & 554.00 & 6.60 \\
\hline $\mathrm{F}$ & 413.62 & 58.57 & 4.68 & 122.50 & 194.70 & 544.30 & 6.49 \\
\hline G & 413.60 & 59.00 & 4.71 & 122.60 & 194.60 & 534.50 & 6.21 \\
\hline $\mathrm{H}$ & 413.50 & 56.77 & 4.77 & 122.60 & 194.60 & 525.00 & 5.82 \\
\hline
\end{tabular}

internal forces remain practically constant in all loading cases, except the maximum axial load developed in the lower tension-only members that obtains a maximum divergence of $38.4 \mathrm{kN}$. In these members, the axial force in loading case A increases by $7.5 \%$ compared to loading case $\mathrm{E}$, when the additional pedestrian is considered at mid-span. The relative deformations of the structure are equally significant with a minimum value of $5.38 \mathrm{~cm}$ in loading case $\mathrm{A}$ and a maximum value of $6.60 \mathrm{~cm}$ in loading case $\mathrm{E}$, attributing to a respective increase of $22.68 \%$.

\section{CONCLUSIONS}

Digital platforms of operation may support interdisciplinary design processes through the introduction of computing facilities and numerical methods of analysis within an integral design process. Digital design enables the designers to collaborate, visualize, research and modify structural performance with relatively high accuracy. This mode of operation requires designers to rethink alternative strategies, to achieve open-loop developments in multivariable systems from early conceptual design stages until fabrication. At the same time, finite element analysis enables accurate verification of intermediate observations and decisions taken throughout the design process, as well as optimization in terms of the local and overall structural behaviour. In all cases, interactive numerical analysis loops in support of parametric associative design processes and physical modelling provide further possibilities not only in classical terms of securing structural analysis, material definitions and sizing of elements but also in the optimization of the design, the system and its components as such. Although no generally ideal design process can be defined, an ideal degree of interaction between the modelling environments, which themselves are chosen specific to the project, is rather envisaged by the integral design process presented in the current paper.

In demonstrating the integral design approach, a cable bending-active structure has been conceptualized, developed and numerically verified, attributing to considerations of adaptability and structural responsiveness to imposed external moving loads. Throughout the development, iterations of physical modelling, physics-based simulations and finite element structural analysis, focused on two integrated directions. First, the digital tools have been 
applied for the design, development and evaluation of results aiming at the investigation of the morphology and behaviour outcomes in satisfying given conditions and constrains. Second, the same loop process has been applied for the simulation of the adaptive structural behaviour showing various stages of adaptability in real time. The idea of such an integral development leads towards design solutions through transformability and real-time structural behaviour control, and offers insides for further development of the structure at refined and detailed levels. In particular, in the preliminary design, the adaptive structures curvilinear typology resulted from the elastic properties of its primary members and their stabilization through horizontal interconnecting tension-only members. In the design development, the load-bearing behaviour and the transformability of the structure have been qualitatively investigated and the construction design and manufacturing of the structural members and connections have been conducted to provide high structural modularization and mass customization. In the design evaluation, the structural behaviour under vertical loading has been investigated for different pretension levels of the connecting cables and a pedestrian movement scenario. Furthermore, the integral design approach of structures using bending-active principles as such may provide new possibilities for an integrated conceptualization, development and investigation of technologically intelligent adaptive structural systems that constitute sustainable design solutions.

\section{REFERENCES}

[1] Kontovourkis, O., Computer-generated circulation diagrams, $\mathrm{PhD}$ Thesis, University of Bath, Bath, 2009.

[2] Kontovourkis, O., Phocas, M.C. \& Tryfonos, G., Prototyping of an adaptive structure based on physical conditions. Architectural Computing, 11(2), pp. 203-223, 2013. http://dx.doi.org/10.1260/1478-0771.11.2.205

[3] Khoo, C.K., Salim, F. \& Burry, J., Designing architectural morphing skins with elastic modular systems. Architectural Computing, 4(9), pp. 397-419, 2011. http://dx.doi.org/10.1260/1478-0771.9.4.397

[4] Phocas, M.C., Kontovourkis, O. \& Nicolaou, N., Design concept of a kinetic formactive hybrid envelope structure. Design \& Nature and Ecodynamics, 9(1), pp. 13-30, 2014.

http://dx.doi.org/10.2495/DNE-V9-N1-13-30

[5] Lienhard, J., Bending-active structures. Form finding strategies using elastic deformation in static and kinetic systems and the structural potentials. PhD Thesis, Research Report 36, ed. J. Knippers, Institute of Building Structures and Structural Design, University of Stuttgart, Stuttgart, 2014.

[6] Fleischmann, M., Knippers, J., Lienhard, J., Menges, A. \& Schleicher, S., Material behaviour. Architectural Design, 216, pp. 44-51, 2012. http://dx.doi.org/10.1002/ad.1378

[7] Schinneger, K., Rutzinger, S., Knippers, J. \& Scheible, F., Biomimetic media façade. Thematic pavilion Expo 2012 Yeosu, South Korea. International Adaptive Architecture Conference, Building Centre, London, 2011.

[8] Schlaich, J., Bergermann, R., Boegle, R., Cachola, A. \& Flagge, S.P., Light Structures. Prestel: New York, 2005.

[9] Otto, F., Spannweiten. Verlag Ullstein: West Berlin, 1965.

[10] Ferre, A., Patent Constructions. New Architecture made in Catalonia. Actar: Barcelona, 2007. 
[11] Menges, A., Material computation. Architectural Design, 216, pp. 14-21, 2012. http://dx.doi.org/10.1002/ad.1374

[12] Kontovourkis, O., Physical data computing in adaptive design process. Proceedings of International Conference on Adaptation and Movement in Architecture, ICAMA 2013, eds C. Ripley \& M. Asefi, Ryerson University: Toronto, pp. 224-236, 2013.

[13] Phocas, M.C., Kontovourkis, O. \& Alexandrou, K., The structural design and construction of a cable bending-active structure. Mobile and Rapidly Assembled Structures IV, eds N. Temmerman \& C.A. Brebbia, Section 1: temporary structures and dwellings, WIT Transactions on the Built Environment, 136, pp. 59-70, 2014. http://dx.doi.org/10.2495/mar140051

[14] Ahlquist, S. \& Menges, A., Realizing formal and functional complexity for structurally dynamic systems in rapid computational means: computational methodology based on particle systems for complex tension-active form generation. Advances in Architectural Geometry 2010, eds C. Ceccato, L. Hesselgren, M. Pauly, H. Pottmann \& J. Wallner, Springer: Vienna, pp. 205-220, 2011.

[15] Phocas, M.C., Kontovourkis, O. \& Ioannou, T., Interdisciplinary research based design: the case of a kinetic form-active tensile membrane. Architectural Engineering Technology, 1(2), pp. 1-7, 2012. http://dx.doi.org/10.4172/2168-9717.1000104

[16] Fleischmann, M. \& Menges, A., Physics-based modeling as an alternative approach to geometrical constrain-modeling for the design of elastically-deformable material systems. Digital Physicality|Physical Digitality, 30th eCAADe Conference Proceedings, Czech Technical University in Prague, Prague, Vol. 1, pp. 565-575, 2012.

[17] Phocas, M.C., Kontovourkis, O. \& Alexandrou, K., Design of a controlled cable bending-active structure. Proceedings of International Conference on Adaptation and Movement in Architecture, ICAMA 2013, eds C. Ripley \& M. Asefi, Ryerson University, Toronto, pp. 237-249, 2013. 